Volume 7

Issue 4 -- Women and Cancer

Article 8

$10-23-2020$

\title{
Cole Relaxation Frequency as a Prognostic Parameter for Breast Cancer
}

William D. Gregory

Shahila Mehboob Christie

John Shell

Georges J. Nahhas

Maharaj Singh

Wendy Mikkelson

Follow this and additional works at: https://aah.org/jpcrr

Part of the Diagnosis Commons, Neoplasms Commons, and the Oncology Commons

\section{Recommended Citation}

Gregory WD, Christie SC, Shell J, Nahhas GJ, Singh M, Mikkelson W. Cole relaxation frequency as a prognostic parameter for breast cancer. J Patient Cent Res Rev. 2020;7:343-8. doi: 10.17294/

2330-0698.1794

Published quarterly by Midwest-based health system Advocate Aurora Health and indexed in PubMed Central, the Journal of Patient-Centered Research and Reviews (JPCRR) is an open access, peer-reviewed medical journal focused on disseminating scholarly works devoted to improving patient-centered care practices, health outcomes, and the patient experience. 


\title{
Cole Relaxation Frequency as a Prognostic Parameter for Breast Cancer
}

\author{
William D. Gregory, PhD, ${ }^{1,2}$ Shahila Mehboob Christie, PhD, ${ }^{1}$ John Shell, PhD, ${ }^{1}$ Georges J. Nahhas, \\ $\mathrm{PhD},{ }^{3}$ Maharaj Singh, $\mathrm{PhD}, 4,5$ Wendy Mikkelson, $\mathrm{MD}^{6}$ \\ ${ }^{1}$ NovaScan, Inc., Milwaukee, WI; ${ }^{2}$ Colleges of Engineering and Health Sciences, University of Wisconsin-Milwaukee, \\ Milwaukee, WI; ${ }^{3}$ Department of Psychiatry \& Behavioral Sciences and Hollings Cancer Center Biostatistics Shared \\ Resource, Medical University of South Carolina, Charleston, SC; ${ }^{4}$ Biostatistics, Marquette University School of \\ Dentistry, Milwaukee, WI; ${ }^{5}$ Advocate Aurora Research Institute, Advocate Aurora Health, Milwaukee WI; ${ }^{6}$ Aurora \\ Comprehensive Breast Health Center, Aurora St. Luke's Medical Center, Milwaukee, WI
}

\begin{abstract}
We previously reported successful classification of breast cancer versus benign tissue using the Cole relaxation frequency measured on tissue excised during breast surgery as part of a study at two urban hospitals in the U.S. Midwest. Using that health system's cancer registry, we have discovered retrospectively that outcomes for patients who participated in the initial study can be classified correctly in 3 well-differentiated categories: nonrecurrent (NR); recurrent with no metastasis (RNM); and recurrent with metastasis (RM). As Cole relaxation frequency increases, the classification moves from NR to RNM and finally to RM. Multivariate analysis showed a significant association of "timecancer-free" for all patients in these recurrent categories, with P-values ranging between 0.0001 to 0.0047. Thus, this follow-up report shows the potential feasibility of using Cole relaxation frequency as a prognostic parameter in a larger prospective study. (J Patient Cent Res Rev. 2020;7:343-348.)
\end{abstract}

Keywords Cole relaxation frequency; breast cancer; prognosis; bioelectrical impedance; recurrence

$\longrightarrow$ ancer is a heterogeneous disease with tumors that can include cells harboring distinct molecular signatures that respond differently to the same therapy. ${ }^{1}$ Breast cancer, in particular, tends to be unique to the person. Tumor characteristics and clinical biomarkers such as size, grade, presence of estrogen and progesterone receptors (ER and $\mathrm{PR}$ ), and human epidermal growth factor receptor (HER2/neu) status all have prognostic value, and some have predictive value. However, there is uncertainty about the chemotherapy sensitivity of some ER-positive, HER2-negative patients with breast cancer, as patients with similar clinical pictures and tumor characteristics can have different clinical outcomes. ${ }^{2}$

Genomic assays that measure the expression of several tumor genes have been developed to inform prognosis and guide therapeutic decisions, ${ }^{3}$ but genomic panels tend to

Corresponding author: William D. Gregory, PhD, NovaScan, Inc., 220 W. Merchandise Plaza, Suite 1230, Chicago, IL 60654 (wgregory@novascanllc.com) provide varied results. A specific tumor may stratify to low risk in one assay and intermediate or high risk in another. The OPTIMA feasibility trial in the United Kingdom was designed to validate the use of multiparameter assays to direct chemotherapy decisions in patients with axillary node metastases. Oncotype $\mathrm{Dx}^{4}$ was the primary assay to determine chemotherapy decisions, but 4 other multiparameter assays also were performed on the tumors. A "substantial discordance" was demonstrated in the risk assignments of individual tumors. ${ }^{5}$ Only $31 \%$ of patients were classified as low/intermediate risk by all 5 panels, and only $8 \%$ were designated as high risk by all 5 assays. More than $60 \%$ of patients were categorized as high risk by at least 1 test and also low risk by at least 1 test. ${ }^{5}$ This is dramatic variation between assays for the same patient group. Given these limitations in available prognostic and predictive technologies, research developing alternate prognostic parameters is needed.

Due to its heterogeneity, cancer has multiple molecular pathways that result in malignancy. Investigating malignancy, by determining the effects of increased disorder at the nanoarchitecture level within cells in 
malignant tissue, may be very useful if found to be a universal change. This is what measuring bioelectrical impedance does. Characterization of the bioelectric properties of human tissue was made possible by Cole et al, whose work produced an equivalent circuit to model biological impedance behavior. ${ }^{6}$ This equivalent circuit, embodied in the Cole function, typically models the spectral data with 4 parameters, including the Cole relaxation frequency (CRF). We have previously demonstrated that $\mathrm{CRF}$ is a parameter that can distinguish malignant from benign breast tissue, ${ }^{7,8}$ with the CRF up to 1000 times greater in malignant than in benign tissue.

The objective of this retrospective follow-up study is to determine if there is a correlation between CRF values measured at the time of surgery to long-term outcomes for these patients, using data available in the cancer registry of a large Midwestern health system. Our hypothesis with this study is that the higher the CRF value, the more aggressive the cancer or the more likely it is that the cancer will recur.

\section{METHODS \\ Study Design}

This retrospective study, approved by the relevant institutional review board, follows up on a previous study's patient cohort for which data were collected from 2003 to $2012 .^{7}$ That study included consented patients undergoing excision of a breast abnormality, benign or malignant. For this retrospective study, only patients with malignancies who had follow-up clinical visits available in the cancer registry maintained by Aurora Health Care (Milwaukee, WI) were included (108 of 187 study participants). Of these 108 patients, 84 did not have cancer recur and 24 had recurrence ( 7 with metastasis). All information regarding study design follows that provided in the published report. ${ }^{7}$

\section{Analysis Methods}

The scientific notation for CRF (typically ranging from $1 \times 10^{5} \mathrm{~Hz}$ to $2 \times 10^{6} \mathrm{~Hz}$ for cancer specimens) is cumbersome, so a dimensionless number was created by dividing (ie, normalizing) the CRF by the lowest value indicative of cancer, $1 \times 10^{5} \mathrm{~Hz}$, to yield values from 1 to 20 . We termed this dimensionless parameter the "normalized Cole relaxation frequency" or nCRF. This prespecified range of nCRF values was chosen to include all data for cancerous lesions found in this patient cohort.

Several histologic prognostic indicators were considered for comparison to nCRF. These included the Nottingham Prognostic Index (NPI), ${ }^{9}$ NPI Plus,${ }^{10}$ and tumor grade. ${ }^{11,12}$ Data available from the Aurora Cancer Registry allowed the computation of NPI, a combination of tumor size, node status, and grade. This produced a scale ranging from 2 to 8 ; the higher the scale number, the worse the prognosis. nCRF score ranges from 1 to 20 , thus a comparison to NPI was possible. NPI Plus includes similar clinicopathologic variables as NPI but additionally includes tumor biology such as luminal classes (luminal A, N, and B), basal classes (basal p53 altered and basal p53 normal), and HER2-positive classes (HER2+/ER+ and HER2+/ER-). ${ }^{13}$ Grade is reported in two ways - 1) calculated from a combination of tubule formation, nuclear polymorphism, and mitotic counts reported on a scale of 1 to 9; and 2) in 3 steps as grade I (well-differentiated, score 3-5), grade II (moderately differentiated, score 6-7), and grade III (poorly differentiated, score 8-9). The 3-step differentiation score was the only information available from the cancer registry. Since the Aurora Cancer Registry reported only the differentiation results for grade and not the 1-9 scores directly, and information regarding luminal and basal classes were not available, it was not possible to make a similar comparison of grade and NPI Plus respective to $\mathrm{nCRF}$, as could be done with NPI. However, it was possible to include grade in the multivariate analysis.

In this work only, NPI was compared to nCRF using exceedance averaging, and both NPI and grade were compared using multivariate analysis to investigate performance of other histology-derived parameters in this patient cohort. Adjusted Cox proportional hazards models were used to determine the effect of nCRF versus NPI, as well as nCRF versus grade, on the length of time after surgery that patients remained cancer-free (ie, "time-cancer-free"). Models were adjusted for age, ER, PR, and HER2/neu status. Hazard ratios (HR) and 95\% confidence intervals were reported. Statistical models were performed in SAS 9.4 software (SAS Institute Inc.).

Likelihood ratios ${ }^{14}$ (referred to hereafter as L) were used to compare the probability of one outcome versus another on the basis of nCRF values and, thus, were used to predict the diagnostic power of nCRF.

To allow comparison of nCRF to patient outcomes, the cancer registry supplied the number of days a patient was cancer free after surgery or, occasionally, "never cancer free" (eg, stage IV) based on follow-up clinic visits. The overall average time of follow-up was 8.2 years. To minimize the effects of missing time-cancerfree data (censored), an exceedance average was used to analyze the data. ${ }^{15}$ This average weighted the measured time-cancer-free by 0 (zero) for nCRF values less than a chosen value and by 1 above that value. This average answered the question: What is the expectation of an average time-cancer-free going forward from the chosen 
value of nCRF? Note that this is different from the overall average, which weights all data by 1 .

\section{RESULTS}

Figure 1 is a comparison of the likelihood of recurrence without metastasis (RNM to nonrecurrence (NR), or L(RNM:NR), and likelihood of recurrence with metastasis (RM) compared to NR, or L(RM:NR), plotted against nCRF. Note that these two groups correspond to two different regions of nCRF values that are contiguous with very little overlap. In nCRF range 3-6, the L(RNM:NR) dominates. When nCRF exceeds 6, the L(RM:NR) dominates and increases dramatically as the nCRF rises. This result can be expressed as follows: below nCRF of 3 , there were no recurrent cases; in nCRF of 3 to 6 , there was recurrence without metastasis; above nCRF of 6 , there is recurrence with metastasis.

Figure 2 illustrates a second independent statistical technique for determining the correlation of the groupings NR, RNM, and RM. This is the aforementioned exceedance averaging technique. The green curve is the exceedance average for the nCRF measurements. This figure also shows how the exceedance average for the NPI compares to the nCRF exceedance average. The first distinction between these two curves is that the NPI curve has no sharp demarcations between regions. In fact, below the graph in Figure 2 are some statistical comparisons between specific NPI regions and nCRF regions. Whereas the nCRF regions have very small comparative P-values (indicating that they are statistically different), the NPI regions NPI-1 and NPI-2 are statistically the same and only region NPI-3 is statistically independent. One can see this graphically in the curve by noting that NPI-1 and NPI-2 fit very well to a straight line while the NPI-3 region bows above that line and is clearly different in the figure. This result means that NPI only distinguishes between metastatic cases and does not further distinguish between nonrecurrence and recurrence with no metastasis. On the other hand, all three regions of nCRF (ie, NR, RNM, and $\mathrm{RM})$ are clearly statistically significantly different.

Multivariate analysis of time-cancer-free showed nCRF was the only parameter to consistently yield correct prognoses for all recurrences, with or without metastasis (Table 1). Adjusting for NPI, age, ER, PR, and HER2/neu, nCRF was significantly associated with higher time-cancer-free among those with combined recurrence (HR: 1.08; $\mathrm{P}=0.0095$ ) and those with metastatic recurrence (HR: $1.18 ; \mathrm{P}=0.0003$ ). $\mathrm{HR}$ was proportionally constant over time, and HR for relevant variables was independent of time. Adjusting for grade, age, ER, PR, and HER2/neu, nCRF was significantly associated with time-cancer-free among those with combined recurrences (HR: $1.083 ; \mathrm{P}=0.0047$ ) and those with metastatic recurrence (HR: $1.185 ; \mathrm{P}=0.0001)$.

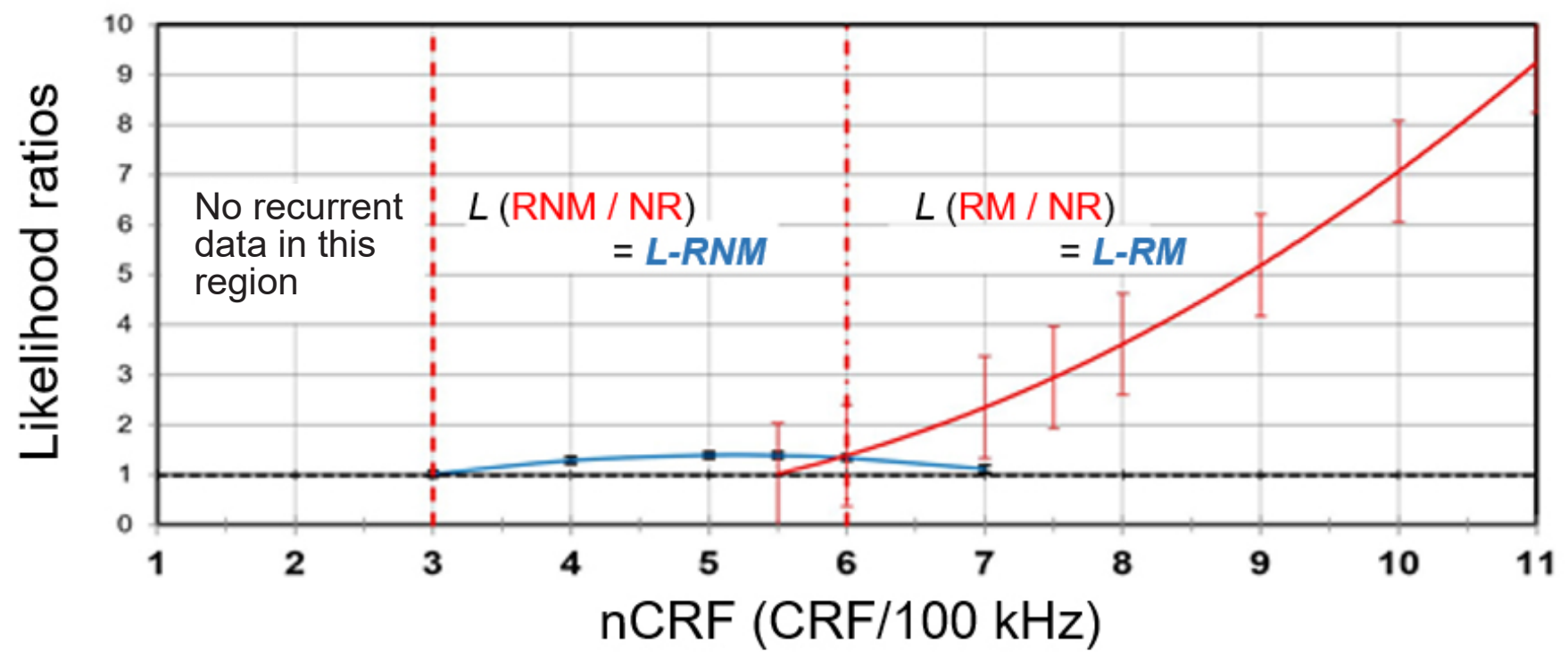

RNM

RM

Figure 1. Likelihood regions obtained from a quadratic fit of likelihood (L) data vs normalized Cole relaxation frequency ( $n C R F)$. Three regions are identified: no recurrence (L-NR) with $n C R F$ of $<3$; recurrence with no metastasis (L-RNM) with nCRF of 3-6; recurrence with metastasis (L-RM) with $n C R F$ of $>6$. The standard error bars for the L-RNM region (plotted in blue) are very small compared to the L-RM region (plotted in red) due to a smaller number of data points in the L-RM region. 


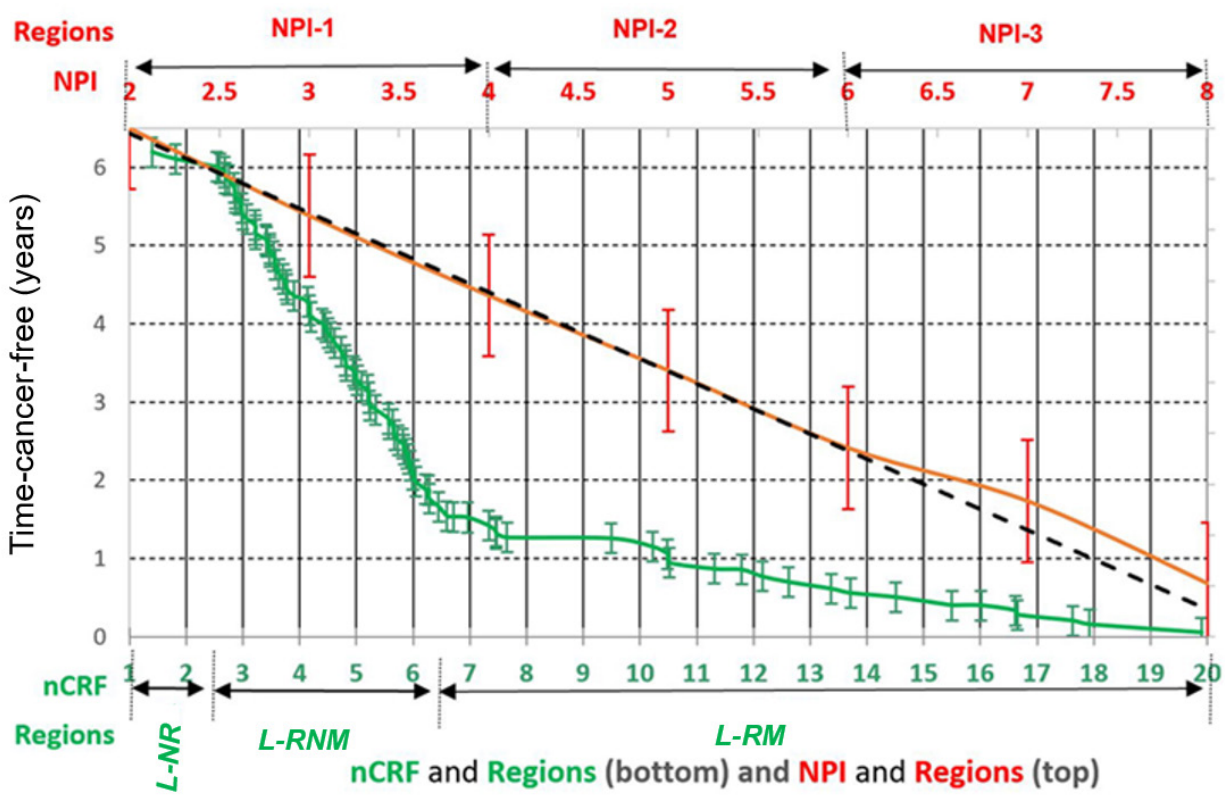

T.TEST Comparisons of regions within NPI and nCRF plots

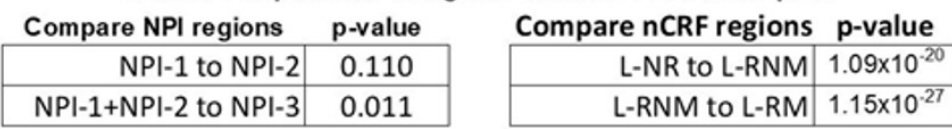

Figure 2. This plot compares exceedance averaged timecancer-free for normalized Cole relaxation frequency ( $n C R F$, in green) and Nottingham Prognostic Index (NPI, in red). The prognosis for time-cancer-free (years) is plotted on the vertical axis. The slope of the nCRF plot changes abruptly at two region boundaries - where L-NR changes to $L-R N M$ and where L-RNM changes to L-RM. NPI plots do not have similar demarcations at the boundary of regions NPI-1 to NPI-2, but there is a change of slope at NPI-2 to NPI-3. The t-test for independence for these regions is displayed below the figure and shows that NPI is only a prognostic parameter for recurrence with metastasis, while nCRF distinguishes all regions, one from the other, allowing statistically significant discrimination of no recurrence (NR), recurrence with no metastasis (RNM), and recurrence with metastasis (RM).

\section{DISCUSSION}

This study found a strong correlation between breast cancer nCRF values obtained at the time of surgery and patients' outcomes of recurrence or time-cancer-free. We found that when nCRF is less than 3, it is likely that the sample, while originally malignant, will not recur. When nCRF is in the range of 3 to 6 , it is likely that the cancer will recur but will not metastasize. However, when $\mathrm{nCRF}$ is greater than 6 , there is an increasingly greater likelihood that the cancer will recur and will metastasize. Multivariate analysis (Table 1) also confirms these conclusions. A larger prospective trial will be needed to compare nCRF and patient outcomes against a more inclusive prognostic group, such as luminal, basal, and HER2/neu subgroups, as is used in NPI Plus.

Our findings are not surprising because bioelectrical impedance appears to measure changes in cells, as they progress from benign to malignant, that are not always discernible by histology. A higher nCRF value may reflect a more extensive change and risk of an aggressive cancer that is prone to recur or metastasize. One theoretical explanation for this is that as cells transform from benign to malignant, they are increasingly disorganized, based on partial wave optical scattering data for cells from the colon, pancreas, and lung. ${ }^{16}$ This disorganization causes electrical polarization of cell contents to decrease, which then results in an increase in the relaxation frequency. ${ }^{7}$

Further, based on the field carcinogenesis model, ${ }^{17}$ this may well be a universal property of cells as they transform regardless of organ of origin of the cell. Evidence of disorganization and/or increased relaxation frequencies has been reported for different cell types besides breast tissue, including colon, pancreas, lung, ${ }^{16}$ and nonmelanoma skin cancer. ${ }^{18}$ Therefore, we hypothesize that nCRF measures a universal characteristic of cancer and malignancy that correlates with the degree that a field of cells has transformed from benign to cancerous. nCRF could be of significant generalized importance for cancer diagnosis and treatment, as it appears to be agnostic of cancer type.

Our observations demonstrate that nCRF values correlate well with breast cancer outcomes of recurrence, metastasis, and time-cancer-free. Multivariate analysis shows nCRF values are statistically significant in contrast to many other prognostic parameters in use today. nCRF is a potentially clinically useful prognostic parameter for breast cancer. This is a small retrospective feasibility study and must be validated in a larger prospective trial that compares $\mathrm{nCRF}$ values with other prognostic parameters and tumor genetic markers. 


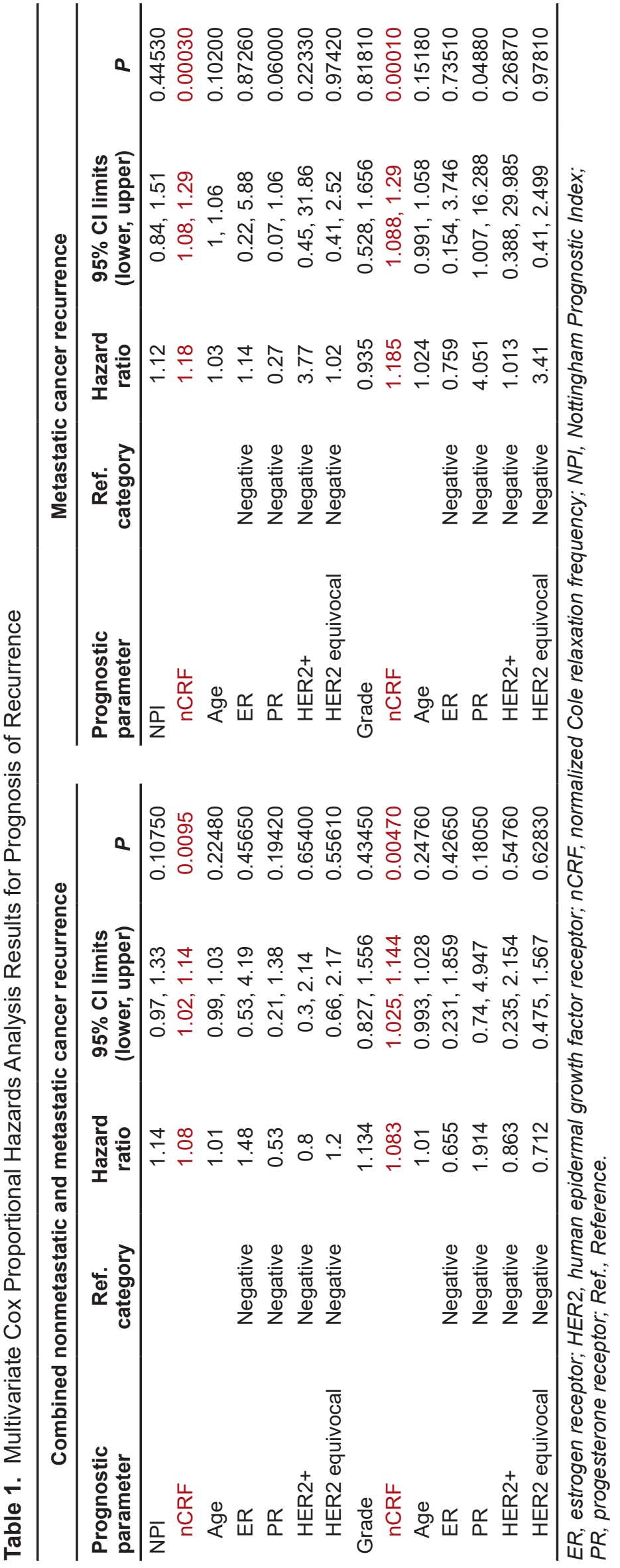

\section{Patient-Friendly Recap \\ - Breast cancer treatment is difficult to predict, as patients with similar clinical and tumor characteristics can have vastly different clinical outcomes. More reliable measures are needed to know which patients may benefit from specific therapies. \\ - The authors studied how electrical current interacts with tissue and derived a parameter called the Cole relaxation frequency to test the feasibility of using this frequency to predict the likelihood a patient's cancer will recur following treatment. \\ - They found that a Cole relaxation frequency level higher than 6 resulted in significantly more patients recurring with metastatic cancer. More research is needed to validate this biomarker as prognostic for breast cancer.}

\section{Acknowledgments}

The study utilizes data from a previous institutional review board-approved study conducted in 2003-2012. Biospecimens were provided through the auspices of the Advocate Aurora Research Institute's Biorepository and Specimen Resource Center (BSRC), and data were provided from the Aurora Cancer Registry. Special thanks to research coordinator Brittany Last of BSRC for her assistance in accessing and organizing cancer registry data.

\section{Author Contributions}

Study design: Gregory, Shell, Mikkelson. Data acquisition or analysis: Gregory, Shell, Nahhas, Singh. Manuscript drafting: Gregory, Christie, Mikkelson. Critical revision: Gregory, Christie, Mikkelson

\section{Conflicts of Interest}

William Gregory and Shahila Mehboob Christie are employed at NovaScan, Inc., and Gregory and John Shell are stockholders in the company. NovaScan has patents pending on technology related to the research reported here. Advocate Aurora Health is a stockholder in NovaScan, but Wendy Mikkelson has no financial interest in the company. The remaining authors (Maharaj Singh and Georges Nahhas) have no conflicts of interest to report.

\section{Funding Sources}

Research funding has been received from the National Science Foundation (Small Business Innovation Research Phase I \#0944454 and Phase IIa and IIb \#1058413). This work was supported in part by Biostatistics Shared Resource (P30 CA138313) of the Medical University of South Carolina's Hollings Cancer Center (Charleston, SC). 


\section{References}

1. Dagogo-Jack I, Shaw AT. Tumour heterogeneity and resistance to cancer therapies. Nat Rev Clin Oncol. 2018;15:81-94. Crossref

2. Yersal O, Barutca S. Biological subtypes of breast cancer: prognostic and therapeutic implications. World J Clin Oncol. 2014;5:412-24. Crossref

3. Paik S, Tang G, Shak S, et al. Gene expression and benefit of chemotherapy in women with node-negative, estrogen receptor-positive breast cancer. J Clin Oncol. 2006;24:3726-34. Crossref

4. McVeigh TP, Kerin MJ. Clinical use of the Oncotype DX genomic test to guide treatment decisions for patients with invasive breast cancer. Breast Cancer (Dove Med Press). 2017;9:393-400. Crossref

5. Stein RC, Dunn JA, Bartlett JM, et al. OPTIMA prelim: a randomised feasibility study of personalised care in the treatment of women with early breast cancer. Health Technol Assess. 2016;20(10):xxiii-xxix, 1-201. Crossref

6. Cole KS, Curtis, HJ. Electrical physiology: electrical resistance and impedance of cells and tissues. In: Otto Glasser (ed). Medical Physics, Volume 1. Yearbook Publishers, Inc.; 1944, pp. 344-8.

7. Gregory WD, Marx JJ, Gregory CW, Mikkelson WM, Tjoe JA, Shell J. The Cole relaxation frequency as a parameter to identify cancer in breast tissue. Med Phys. 2012;39:4167-74. Crossref

8. Shell J, Gregory WD. Efficient cancer detection using multiple neural networks. IEEE J Transl Eng Health Med. 2017;5:2800607. Crossref

9. Haybittle JL, Blamey RW, Elston CW, et al. A prognostic index in primary breast cancer. Br J Cancer. 1982;45:361-6. Crossref

10. Green AR, Soria D, Stephen J, et al. Nottingham Prognostic Index Plus: validation of a clinical decision making tool in breast cancer in an independent series. J Pathol Clin Res. 2016;2:32-40. Crossref
11. Elston CW, Ellis IO. Pathological prognostic factors in breast cancer. I. The value of histological grade in breast cancer: experience from a large study with long-term follow-up. Histopathology. 1991;19:403-10. Crossref

12. Rakha EA, Reis-Filho JS, Baehner F, et al. Breast cancer prognostic classification in the molecular era: the role of histological grade. Breast Cancer Res. 2010;12(4):207. Crossref

13. Green AR, Soria D, Powe DG, et al. Nottingham prognostic index plus (NPI + ) predicts risk of distant metastases in primary breast cancer. Breast Cancer Res Treat. 2016;157:65-75. Crossref

14. Centre for Evidence-Based Medicine, University of Oxford. Likelihood ratios. Accessed August 13, 2020. https://www. cebm.ox.ac.uk/resources/ebm-tools/likelihood-ratios

15. Park, KI. Fundamentals of Probability and Stochastic Processes with Applications to Communications. Springer International Publishing; 2018. Crossref

16. Subramanian H, Roy HK, Pradhan P, et al. Nanoscale cellular changes in field carcinogenesis detected by partial wave spectroscopy. Cancer Res. 2009;69:5357-63. Crossref

17. Dakubo GD, Jakupciak JP, Birch-Machin MA, Parr RL. Clinical implications and utility of field cancerization. Cancer Cell Int. 2007;7:2. Crossref

18. Svoboda RM, Gharia MJ, Shell J, Gregory WD. Bioimpedance measurement as an assessment of margin positivity in Mohs surgical specimens of nonmelanoma skin cancer: management implications. J Am Acad Dermatol. 2018;79:591-3. Crossref

(C) 2020 Advocate Aurora Health, Inc. 